\title{
Crónicas de la expansión. Notas sobre el XIV Congreso de Ciencia Política
}

Chronicles of the Expansion. Notes On the XIV Political Science Conference

\author{
PATRICIO G. TALAVERA \\ Universidad de Buenos Aires, Argentina \\ Universidad Nacional de San Martín, Argentina \\ ptalavera@sociales.uba.ar
}

\section{Introducción}

Durante los días que fueron desde el 17 hasta el 20 de julio de 2019 se desarrolló en el campus de la Universidad Nacional de San Martín(UNSAM), el XIV Congreso de Ciencia Política de la Sociedad Argentina de Análisis Político (SAAP). Esta nota se sostendrá sobre dos ejes: en primer lugar, un análisis de la participación en las diferentes actividades y, en segundo lugar, un recorrido sobre los paneles y simposios ofertados durante esos días.

Desde la gestación del primer Congreso, en Huerta Grande, provincia de Córdoba, en 1993, el espíritu de los Congresos de la SAAP ha sido evidente: el sentido federal al momento de definir las plazas donde desarrollar cada dos años su máximo evento. Siempre basculando en la tensión entre Buenos Aires y el interior nacional, los Congresos de la SAAP supieron mantener un equilibrio entre las distintas regiones del país, como marca la Tabla 1: siete fueron organizados en la cuenca mediterránea del país, 5 se realizaron en la Provincia de Buenos Aires y la Ciudad Autónoma de Buenos Aires, y dos en Cuyo. De esta manera, el enlace entre la agenda nacional y las problemáticas locales de la disciplina se ha demostrado presente en facilitar los intercambios y enriquecer los debates. Al cabo de 26 años, como bien ha destacado en su momento Rotman $(2010 ; 2012)$, el Congreso de la SAAP ha combinado dos elementos no siempre presentes en las citas académicas regionales. En primer lugar, la constitución de un foro de referencia nacional e internacional, siendo objeto de interés por parte de especialistas de las universidades mejor rankeadas del mundo. En segundo lugar, se ha constituido como elemento de cohesión de las distintas agendas regionales que, yuxtapuestas, articulan las principales preocupaciones a lo largo y ancho del país, forjando un robusto proceso de consolidación institucional (Bulcourf y D’Alessandro, 2002; Abal Medina, Leiras y D'Alessandro, 2005). 
TABLA 1

Congresos Nacionales de Ciencia Política organizados por la Sociedad Argentina de Análisis Político (SAAP)

\begin{tabular}{|c|c|c|c|}
\hline Número & Año & Ciudad & Título \\
\hline $\mathbf{I}$ & 1993 & Huerta Grande & El malestar en la democracia \\
\hline II & 1995 & Mendoza & Globalización, entre el conflicto y la integración \\
\hline III & 1997 & Mar del Plata & Democracia, reforma económica y cuestión social \\
\hline IV & 1999 & Buenos Aires & $\begin{array}{l}\text { Desempeño institucional y control democrático a } \\
\text { fines de siglo }\end{array}$ \\
\hline $\mathbf{V}$ & 2001 & Río Cuarto & $\begin{array}{l}\text { La primacía de la política. Ética y responsabilidad } \\
\text { de los actores sociales y políticos }\end{array}$ \\
\hline VI & 2003 & Rosario & $\begin{array}{l}\text { La política en un mundo incierto: representación, } \\
\text { gobernabilidad democrática e inclusión social }\end{array}$ \\
\hline VII & 2005 & Córdoba & Agendas regionales en escenarios de conflicto \\
\hline VIII & 2007 & Buenos Aires & $\begin{array}{l}\text { ¿Hacia dónde va la Argentina? Inserción } \\
\text { internacional, calidad institucional y nuevas } \\
\text { representaciones }\end{array}$ \\
\hline IX & 2009 & Santa Fe & $\begin{array}{l}\text { Centros y periferias: equilibrios y asimetrías en las } \\
\text { relaciones de poder }\end{array}$ \\
\hline $\mathbf{X}$ & 2011 & Córdoba & $\begin{array}{l}\text { Democracia, integración y crisis en el nuevo orden } \\
\text { global: tensiones y desafíos para el análisis político }\end{array}$ \\
\hline XI & 2013 & Paraná & $\begin{array}{l}\text { La política en movimiento. Estados, democracia y } \\
\text { diversidades regionales }\end{array}$ \\
\hline XII & 2015 & Mendoza & $\begin{array}{l}\text { La política en balance. Debates y desafíos } \\
\text { regionales }\end{array}$ \\
\hline XIII & 2017 & Buenos Aires & $\begin{array}{l}\text { La política en entredicho. Volatilidad global, } \\
\text { desigualdades persistentes y gobernabilidad } \\
\text { democrática }\end{array}$ \\
\hline XIV & 2019 & San Martín & $\begin{array}{l}\text { La política en incertidumbre. Reordenamientos } \\
\text { globales, realineamientos domésticos y la cuestión } \\
\text { de la transparencia }\end{array}$ \\
\hline
\end{tabular}

Fuente: Elaboración Propia en base a Gervasoni (1997, 1999 y 2002), De Luca (2006), Rotman (2010 y 2012), Cruz (2013) y SAAP (www.saap.org.ar). 
El Congreso contó durante las cuatro jornadas de su desarrollo con la participación de autores e investigadores de primer nivel de ámbito nacional. En la ceremonia inicial, se hizo presente la Vicepresidenta de la Nación, Gabriela Michetti, el Vicegobernador de la provincia de Buenos Aires, Daniel Salvador, el Intendente del partido de San Martín, Gabriel Katopodis y otras autoridades nacionales y provinciales, lo que resalta el reconocimiento de distintos niveles gubernamentales a los Congresos organizados por SAAP. El encuentro también contó con una gran cantidad de investigadores argentinos reconocidos internacionalmente: desde Flavia Freidenberg (Universidad Nacional Autónoma de México), Hugo Quiroga (Universidad Nacional de Rosario) y Carlos Acuña (Universidad Nacional de San Martín), pasando por Ernesto Calvo (Maryland University), Andrés Malamud (Universidade de Lisboa), María Esperanza Casullo (Universidad Nacional de Río Negro), Estela Miranda, (Universidad Nacional de Córdoba) y Mario Riorda (Universidad Austral), hasta llegar a referentes históricos como Mabel Thwaites Rey e Isidoro Cheresky, ambos de la Universidad de Buenos Aires. En consonancia con la presencia de dichos referentes, pero también, con tendencias mundiales en materia de agenda de investigación, el XIV Congreso de la SAAP dividió sus presentaciones en nueve mesas temáticas. En esa división se incluyeron referencias a agendas de relevancia, como Género y Relaciones Internacionales, pero sin dejar de abarcar áreas de una densidad y envergadura histórica en la generación de aportaciones científicas a la disciplina. De esta manera se incluyeron áreas como Teoría y Filosofía Política; Estado, Administración y Políticas Públicas; Opinión Pública y Marketing Político; Política Comparada; Instituciones Políticas; Historia y Política; y Desarrollo, Enseñanza y Metodología de la Ciencia Política. Todas ellas reconocieron en su interior entre 8 y 19 sub-áreas temáticas, lo que da muestra de la complejidad, diversidad y proliferación de la Ciencia Política en la Argentina de hoy. En paralelo, se organizaron mesas especiales con presencia de dirigentes políticos de todos los sectores y partidos de Argentina. Esto último viene a resaltar un diálogo fluido con la política como actividad y objeto de investigación, por un lado, y la persistente pluralidad política de la SAAP, por el otro.

Sin embargo, dicha complejidad no quedó desacompasada de la estructura funcional del Congreso, el cual, fue incorporando en forma de simposios, mecanismos de interacción de experiencias sobre temáticas que hacían a la coyuntura de agenda de investigación. En 2019, éstos crecieron hasta ser 14 eventos perfectamente distinguibles del resto del cuerpo del Congreso. En este tipo de actividad se desarrollan temas con una clara raigambre y popularidad en la academia local e internacional: Estudios Electorales, Política Latinoamericana Comparada, Justicia y Política, y Reforma Política y Observación Electoral. A estos focos de atención más clásicos, se 
adosaron temáticas fundamentales de coyuntura que congregan a un creciente número de investigadores. La variedad caracterizó la estructuración de los simposios: por citar ejemplos, el organizado en torno a los diez años de la Asignación Universal por Hijo; el de Políticas Públicas de Seguridad; el de Ambiente; Comunicación Política, Regionalismo e Integración, Partidos Políticos; Desigualdad Juvenil; Sociología Política; Economía Comparada; y finalmente, Movilización Política. Toda una carta de temas desarrollados con intensidad, y en muchos casos, bajo formas de cooperación con unidades académicas del exterior, que dan cuenta de la prosperidad institucional, flexible y adaptativa, de la Ciencia Política argentina.

Un lugar de distinción en esa tarea de expansión fronteriza de las posibilidades de la disciplina tiene sin duda la consolidación de los premios por área que se entregan en cada edición. Se trata de la construcción de estímulos para los investigadores recientemente egresados, a manera de alentarlos en la formación de perfiles especializados, que abarcan desde Teoría Política y Género hasta Instituciones y Política Comparada. Todo ello en un proceso de evaluación de méritos en el que participan los invitados con mayor trayectoria del Congreso en calidad de jurados, sin conocer la identidad de los postulantes. Este proceso alimenta una renovación que fortalece la integración institucional de la disciplina en todo el país. Con el paso del tiempo ha aumentado en forma relevante la cantidad de premios: en el X Congreso se otorgaron siete premios; en este, los reconocimientos fueron once.

Otro elemento fundamental para rescatar y valorar como síntoma de consolidación de los Congresos como espacios de construcción del tejido disciplinario es la proliferación de presentaciones de libros. En muchos casos, si bien aún no han salido al mercado editorial, los autores deciden presentarlos como "conferencias-avances". Esto subraya dos elementos interesantes: el primero, la valoración del Congreso como ámbito de referencia a la hora de presentar ante colegas los frutos finales de investigaciones relevantes de larga duración; el segundo, la relevancia adquirida por estos ámbitos de encuentro como parte de los circuitos no oficiales de difusión editorial. De esta manera, el XIV Congreso SAAP fue escenario de la presentación de investigaciones. por ejemplo, en el ámbito del análisis del clientelismo político, es mencionable "Non-Policy Politics: Richer Voters, Poorer Voters and the Diversification of Electoral Strategies" de María Victoria Murillo (Columbia University) y Ernesto Calvo (Maryland University); la cual fue acompañado por los comentarios de Andrés Malamud (Universidad de Lisboa), María Esperanza Casullo (Universidad Nacional de Rio Negro) y Virginia Oliveros (Tulane University). En cuanto a investigaciones sobre Instituciones Políticas, también recibió gran atención la promoción del libro "Understanding Institutional Weakness: Power and Design in Latin American Institutions", de Steven Levitsky (Harvard University), María Victo- 
ria Murillo (Columbia University) y Daniel Brinks (University of Texas), en cuya presentación fue moderador el presidente de la SAAP, Martín D’Alessandro, con comentaristas como Marcelo Leiras (Universidad de San Andrés) y Jacqueline Behrend (Universidad de San Martín). Las temáticas de los libros presentados, lejos de establecer una preferencia excluyente y concentrar los énfasis en una rama o agenda de investigación, abarcaron ámbitos tan plurales como en crecimiento. Las presentaciones de libros, que llegaron a ser 21 en este Congreso, tocaron temas como la evolución metodológica en la enseñanza universitaria de la Ciencia Política ("La enseñanza de la Ciencia Política argentina" de Martín D'Alessandro perteneciente a la Universidad de Buenos Aires y Diego Gantus proveniente de la Universidad Nacional de Rosario), cuestiones de coyuntura económica ("Debajo del agua" del senador Martín Lousteau) hasta cuestiones más vinculadas a la sociología política ("Soberanía ciudadana y presidentes en busca de hegemonía", de Isidoro Cheresky de la Universidad de Buenos Aires, con los comentarios de Alejandro Grimson y Alejandro Katz, ambos de la Universidad Nacional de San Martín).

Este nivel de actividad se tradujo en una cantidad de paneles destacable: de los 598 en esta edición 435 fueron paneles regulares, vinculados a la distribución temática arriba descripta y que atrajeron a ponentes de todo el país en relación a los múltiples temas de investigación propuestos. Esa diversidad no impidió la jerarquización temática y el ordenamiento disciplinario necesario para darle un lugar visible a cada especialización. El Congreso contó con 73 mesas especiales de temas específicamente escogidos por su relevancia y proyección futura. La coordinación de grandes actividades, el establecimiento de lazos de cooperación interinstitucional, la constitución de redes internacionales con universidades de América Latina en particular, pero también del resto del mundo, tuvo su lugar de operación en el Congreso. De esta manera, se constituyeron encuentros de directivos de carreras de Ciencia Política, y dos sesiones plenarias especiales. En paralelo, se desplegaron 51 paneles de estudiantes, para dar cauce a nuevas generaciones de investigadores que puedan proponer sus intereses y nutrir su trabajo con críticas de académicos de trayectoria. La articulación de esos paneles también interactuó con doce conferencias magistrales, en las cuales autores e investigadores de todo el mundo visitaron el Congreso de SAAP conformando así un cuerpo de 44 invitados internacionales de gran prestigio. Por otra parte, se llevaron adelante paneles de homenaje a profesores fallecidos, como Luis Aznar y Mario Serrafero. Cabe destacar también el desarrollo exitoso de novedosos paneles de frontera, en colaboración con otras entidades, bajo el título «La Ciencia Política y las neurociencias en los desafíos de la Argentina del futuro».

Haciendo foco en protestas sociales y movimientos políticos, el Congreso organizó tres eventos con Donatella Della Porta (Scuola Normale Superiore de Pisa); la cuestión del devenir de los desarrollos profesionales en el sector 
público se enriqueció con la intervención de Michael Barzelay (London School of Economics), coordinado por el profesor Luciano Andrenacci (Universidad Nacional de San Martín); los modelos de sistemas de comunicación y su impacto en las campañas electorales, fueron analizados por Christina HoltzBacha (Friedrich-Alexander Universitat) y Adriana Amado (Universidad Argentina de la Empresa-Universidad de Buenos Aires). Una miríada de expertos internacionales especialmente invitados disertaron en el Congreso: Murat Yulek (Istambul Commerce University), Paulo Ravecca (Universidad de la República), Eric Belanguer (McGill University), Daniel Buquet (Universidad de la República), Nicolas Somma (Pontificia Universidad Católica de Chile), Kenneth Roberts (Cornell University), Fuguo Cao (Central University of Finance and Economics of China), Charles Jones (Cambridge University), Maritza Paredes (Pontificia Universidad Católica del Perú), Oswaldo do Amaral (Universidad Estatal de Campinas), Juan Pablo Luna (Pontificia Universidad Católica de Chile), Angelica Cuellar (Universidad Nacional Autónoma de México), Denise Merklen (Sorbonne Nouvelle Paris III), Mauricio Domingues (Universidade do Estado do Rio de Janeiro), Filipe Teles (Universidade de Aveiro), Lorenzo Córdova (Instituto Nacional Electoral, de México), Octavio Amorim Neto (Fundação Getúlio Vargas), Celina Souza (Universidade Federal da Bahia), Renato Boschi (Universidade do Estado do Rio de Janeiro), Fernando Mayorga (Universidad Mayor San Simón), Silvana Krause (Universidade Federal do Rio Grande do Sul), Richard Snyder (Brown University), Roderick Hart (University of Texas at Austin), Janina Onuki.

\section{TABLA 2}

Estadística sobre composición y participación en el XIV Congreso de la SAAP

\begin{tabular}{|c|c|c|c|c|c|c|c|c|c|c|c|c|c|c|}
\hline Congreso & $\begin{array}{c}\text { XIII } \\
1993\end{array}$ & $\begin{array}{l}\text { XIV } \\
1995\end{array}$ & $\begin{array}{c}\text { XI } \\
1997\end{array}$ & $\begin{array}{c}\text { XIII } \\
1999\end{array}$ & $\begin{array}{r}\text { XIII } \\
2001\end{array}$ & $\begin{array}{c}\text { XIV } \\
2003\end{array}$ & $\underset{2005}{\text { XI }}$ & $\begin{array}{c}\text { XII } \\
2007\end{array}$ & $\begin{array}{r}\text { XIII } \\
2009\end{array}$ & $\begin{array}{l}\text { XIV } \\
2011\end{array}$ & $\underset{2013}{\mathbf{X I}}$ & $\begin{array}{c}\text { XIII } \\
2015\end{array}$ & $\begin{array}{l}\text { XIII } \\
2017\end{array}$ & $\begin{array}{c}\text { XIN } \\
2019\end{array}$ \\
\hline Cantidad de inscriptos & 400 & 900 & 792 & 603 & 509 & 1170 & 981 & 953 & 1239 & 1718 & 1830 & 2036 & 2326 & 2035 \\
\hline Paneles regulares & 25 & 34 & 23 & 24 & 27 & 40 & 41 & 49 & 98 & 128 & 290 & 273 & 259 & 435 \\
\hline Mesas especiales & 3 & 8 & 8 & 12 & 6 & 21 & 17 & 24 & 60 & 33 & 22 & 51 & 34 & 73 \\
\hline Conferencias & 0 & 1 & 2 & 6 & 2 & 1 & 2 & 2 & 4 & 21 & 15 & 5 & 8 & 12 \\
\hline Sesiones plenarias & 1 & 2 & 2 & 2 & 3 & 4 & 3 & 3 & 3 & 3 & 4 & 3 & 2 & 2 \\
\hline Simposios & $s / d$ & $s / d$ & $5 / d$ & $s / d$ & $5 / d$ & $s / d$ & $s / d$ & $5 / d$ & $s / d$ & 1 & 10 & 11 & 12 & 14 \\
\hline Presentaciones de ibros & $s / d$ & 1 & $s / d$ & $s / d$ & $s / d$ & 3 & 0 & 3 & 23 & 29 & 43 & 22 & 25 & 21 \\
\hline Paneles de estudiantes & 0 & $s / d$ & 0 & 0 & 4 & 6 & 8 & 11 & 19 & 32 & 31 & 42 & 35 & 51 \\
\hline
\end{tabular}

Fuente: Elaboración Propia en base a Gervasoni (1997, 1999 y 2002), De Luca (2006), Rotman (2010 y 2012), Cruz (2013) y SAAP (www.saap.org.ar).(Universidad de San Pablo), Flávia Biroli (Asociación Brasileña de Ciencia Política), Pierre Ostiguy (Universidad Católica de Córdoba), entre otras figuras de relieve. 
El interés que suscitó el Congreso se reveló en las solicitudes de inscripción, que en esta ocasión se elevaron hasta alcanzar los 3.297 solicitantes que terminaron materializándose en 2.035 asistentes efectivos. El escenario más factible es que el número de asistentes concretos haya sido mayor al registrado, dado que, a diferencia del Congreso del año 2017, era posible, por las dimensiones del campus que albergó a las actividades, ingresar sin acreditarse. Sobre los asistentes registrados, casi la mitad fue ponente o comentarista: 912 acreditados. Una porción menor pero muy relevante participó además en su condición de socio SAAP: 564 personas a lo largo de los cuatro días. La asistencia, por otra parte, mostró como la misma Universidad Nacional de San Martín y la Universidad de Buenos Aires mantuvieron un rol preponderante en la convocatoria: casi 80 asistentes acreditados tenían a la universidad anfitriona como filiación académica, mientras que 153 reconocían su pertenencia a la Universidad de Buenos Aires. La resonancia del evento también se percibió en el número de asistentes extranjeros, que alcanzó las 272 personas.

Sensible a cuestiones de agenda y actualidad en eventos, paneles y demás propuestas del Congreso, la SAAP mantuvo su compromiso con la cuestión de género. En la web tanto del Congreso como de la misma SAAP, se difundió e invitó a la participación de una encuesta sobre violencia y género en Ciencia Política, en procura de obtener información empírica sobre esta problemática, por primera vez en la historia de la disciplina, la cual fue contestada por más de 700 colegas.

El Congreso por otra parte, contó con un cierre de gran asistencia, que supuso un balance del esfuerzo institucional y las oportunidades generadas, a cargo del Dr. Martín D’Alessandro como Presidente de SAAP, de la Dra. María Matilde Ollier en calidad de Decana de la Escuela de Política y Gobierno de la Universidad Nacional de San Martín, y del Intendente de San Martín, Gabriel Katopodis.

\section{Conclusión}

Al cabo de un cuarto de siglo, el desarrollo de la Ciencia Política en Argentina reconoce una nueva expansión de sus fronteras. En este sentido caben señalar cinco vectores fundamentales que alimentan dicha expansión: 1) el reconocimiento externo; 2) la creación de redes; 3) la participación de jóvenes investigadores; 4) la recreación de espacios de interlocución con referentes disciplinarios; 5) la presencia institucional de la SAAP.

El reconocimiento externo quedó evidenciado en la gran cantidad de invitados extranjeros, los cuales jerarquizaron al Congreso no solo como 
instancia nacional, sino como referencia regional en la producción académica en Ciencia Política, y como una instancia madura y diversificada en materia de agenda de investigación. En segundo lugar, la reunión de directivos, la participación de mesas con temática internacional y de política comparada, abrió la oportunidad de intercambios con académicos de países vecinos y de comparar experiencias de regiones totalmente disímiles con América Latina, tales como China o el Sudeste asiático, lo que no ocurría con frecuencia en el pasado. Se trata de la internacionalización de una agenda que no deja de perder capilaridad local.

El tercer aspecto, la participación de jóvenes investigadores, tuvo múltiples vertientes y demostraciones. Desde la presencia palpable en los mecanismos de logística y organización del Congreso, hasta los mismos paneles temáticos para la exposición de estudiantes, la línea de continuidad generacional quedó consolidada. La experiencia de investigadores de grado con paneles propios en un Congreso internacional no se encuentra íntegramente incorporada en todas las academias regionales, lo cual refuerza la distinción de la SAAP en su esfuerzo inclusivo.

El cuarto elemento va unido íntimamente con el segundo aspecto: la constitución de conferencias, foros y presentación de libros reforzaron el liderazgo y referencia de históricos autores lo cual facilitó la difusión de relevantes aportes teóricas.

Por último, la dimensión geográfica. Desde su primer Congreso hasta este último, la SAAP se mantuvo sensible a una distribución ecuánime del conocimiento entre las distintas regiones del país, para evitar una macrocefalia en la Ciudad de Buenos Aires. En esta ocasión, producto del desarrollo de nuevas universidades en las últimas décadas en el Gran Buenos Aires, la SAAP alejó nuevamente de la capital del país a la concreción de su cita mayor. Así, se situó en un nuevo polo académico con grandes avances en los últimos 20 años, como la Universidad Nacional de San Martín, la cual muestra un creciente vigor en cantidad de alumnos e incorporación de profesionales nacionales e internacionales. El dotar de integralidad es también construir capilaridad territorial para que la difusión sea lo más extensiva, intensiva, y prolífica posible; de modo tal que el recurso formativo y académico tenga solo el condicionante del mérito personal, sin clivajes geográficos que establezcan preferencias injustas. En otras palabras, lo que se buscó fue un desarrollo compartido como aspiración presente y futura de la Ciencia Política en Argentina. 


\section{Referencias Bibliográficas}

Abal Medina, J., M. Leiras, y M. D’Alessandro (2005). Ciencia política en Argentina: el camino de la institucionalización dentro y fuera de las aulas universitarias. En Revista de Ciencia Política 25 (1).

Bulcourf, P. y M. D'Alessandro (2002). La ciencia política en la Argentina. Desde sus comienzos hasta los años 80. En Revista de Ciencias Sociales - 13, noviembre.

Cruz, F. (2013). La (ciencia) política avanza: El XI Congreso Nacional de Ciencia Política en Paraná. En Revista SAAP, 7 (2).

De Luca, M. (2006). Ciencia política en Córdoba capital: un balance sobre el VII Congreso Nacional de la SAAP. En Revista SAAP, 2 (3).

Gervasoni, C. (1997). El Congreso de Mar del Plata en números. En Boletín SAAP, 3 (5).

Gervasoni, C. (1999). Un balance del Congreso de Buenos Aires. En Boletín SAAP, $5(9)$.

Gervasoni, C. (2002). El V Congreso Nacional en Río Cuarto: datos alentadores en medio de la crisis. En Revista SAAP, 1 (1).

Rotman, S. (2010). Los logros de la ciencia política argentina: el IX Congreso Nacional de la Sociedad Argentina de Análisis Político en Santa Fe. En Revista SAAP, 4 (2).

Rotman, S. (2012). La promesa de la ciencia política: El X Congreso de la Sociedad Argentina de Análisis Político en perspectiva comparada. En Revista SAAP, 6 (2). 